\title{
Bokashi em mudas de Campomanesia adamantium (Cambess.) O. Berg: aspectos morfométricos e fotoquímicos
}

\author{
Cleberton Correia SANTOS ${ }^{1 *}$, Rodrigo da Silva BERNARDES ${ }^{1}$, Ademir GOELZER ${ }^{2}$, \\ Mariana Lescano GEIST ${ }^{1}$, Maria do Carmo VIEIRA ${ }^{1}$, Néstor Antonio Heredia ZÁRATE ${ }^{1}$
}

\author{
${ }^{1}$ Faculdade de Ciências Agrárias, Universidade Federal da Grande Dourados, Dourados, Mato Grosso do Sul, Brasil. \\ ${ }^{2}$ Faculdade de Ciências Biológicas e Ambientais, Universidade Federal da Grande Dourados, Dourados, Mato Grosso do Sul, Brasil. \\ *E-mail: cleber_frs@yahoo.com.br
}

Recebido em maio/2018; Aceito em outubro/2018.

RESUMO: A Campomanesia adamantium (Cambess.) O. Berg (Myrtaceae) é uma espécie nativa do Cerrado, com potencial medicinal e alimentício. Portanto, é necessário seu cultivo ex situ; porém, são incipientes os estudos na fase de crescimento de mudas da espécie com uso de biofertilizantes. Todavia, não há informações sobre a quantidade adequada desse material para mudas da espécie. Assim, objetivou-se conhecer o efeito do bokashi nos aspectos morfométricos e fotoquímicos em mudas de $C$. adamantium. Avaliaram-se cinco doses de bokashi $\left(0,0 ; 5,0 ; 10,0 ; 15,0\right.$ e $20,0 \mathrm{~g} \mathrm{~kg}^{-1}$ de solo), incorporadas a um Latossolo Vermelho Distroférrico, de textura argilosa. $\mathrm{O}$ delineamento experimental utilizado foi em blocos casualizados, com quatro repetições, e 20 mudas por unidade experimental. Houve mortalidade de $100 \%$ das mudas produzidas com $20 \mathrm{~g}$ de bokashi. A sobrevivência e $\mathrm{F}_{\mathrm{v}} / \mathrm{F}_{0}$ reduziram em função de doses crescentes de bokashi. $\mathrm{O}$ diâmetro do coleto máximo foi observado nas mudas cultivadas em solo com adição de $6,48 \mathrm{~g}$ de bokashi. Verificou-se crescimento do CML e LML com doses crescentes de bokashi. A adição de bokashi favorece as características morfométricas, mas acima de $15 \mathrm{~g}$ de bokashi é prejudicial na produção de mudas de $C$. adamantium.

Palavras-chave: biofertilizante, frutífera nativa, planta medicinal, Myrtaceae.

\section{Bokashi on seedlings of Campomanesia adamantium (Cambess.) O. Berg: morphometric and photochemical aspects}

\begin{abstract}
The Campomanesia adamantium (Cambess.) O. Berg (Myrtaceae) is specie native to the 'Cerrado', with medicinal and food potential. Therefore, it's necessary ex situ cultivation; however, the studies in growth phase of seedlings of the species with use of biofertilisers are incipient. However, there isn't information on adequate amount of this material for seedlings of the species. Thus, objective was to know the effect of bokashi on morphometric and photochemical aspects in C. adamantium seedlings. Five doses of bokashi $\left(0.0,5.0,10.0,15.0\right.$ and $20.0 \mathrm{~g} \mathrm{~kg}^{-1}$ soil), added in a Dystroferric Red Latosol, of clay texture, were evaluated. The experimental design was in randomized block, with four replicates, 20 seedlings per experimental unit. There was mortality of $100 \%$ of the seedlings produced with $20 \mathrm{~g}$ bokashi. Survival and $\mathrm{F}_{\mathrm{v}} / \mathrm{F}_{0}$ decreased as a function of increasing doses of bokashi. The maximum collar diameter was observed in seedlings cultivated in soil with addition of $6.48 \mathrm{~g}$ bokashi. There was growth of CML and LML with increasing doses of bokashi. The addition of bokashi favors the morphometric characteristics, but above $15 \mathrm{~g}$ bokashi is harmful in the production of $C$. adamantium seedlings.
\end{abstract}

Keywords: biofertilisers, native fruits, medicinal plant, Myrtaceae.

\section{INTRODUÇÃO}

A Campomanesia adamantium (Cambess) O. Berg (Myrtaceae), também conhecida como guavira é uma espécie arbustiva, frutífera, endêmica do Cerrado (LORENZI et al., 2006). A espécie apresenta importância alimentícia e medicinal, e suas mudas podem ser inseridas em áreas de recuperação, sistemas agroflorestais biodiversos e/ou pomares comerciais, devido a seu valor de agregação.

As folhas e cascas da espécie apresentam efeitos antiinflamatório e antidiarreico (RODRIGUES e CARVALHO, 2001), antiúlcera péptica (COELHO et al., 2004), antisséptico das vias urinárias (LORENZI et al., 2006), antiviral, antiulcerogênico, citotóxico, antihepatotóxico, antihipertensivo, hipolipidêmica, anti-inflamatório, antiplaquetário (MACHADO et al., 2008), e antiproliferativo
(PASCOAL et al., 2014). Do ponto de vista alimentício, seus frutos são adocicados, e a casca e polpa apresentam-se como fonte de vitamina $\mathrm{C}$ e compostos antioxidantes (PEREIRA et al., 2012), garantindo a bioatividade funcional na alimentação humana. Considerando essas informações, torna-se imprescindível estabelecer tratos culturais almejando seu cultivo ex situ.

Para o cultivo de plantas medicinais nativas, o uso de biofertilizantes pode favorecer a qualidade das mudas, uma vez que, quando adicionados ao substrato ou solo de cultivo, melhoram os atributos químicos, físicos e microbiológicos (HIGASHIKAWA et al., 2010; ZACCARDELLI et al., 2013). O bokashi é um biofertilizante fermentado por microorganismos benéficos que favorecem a mineralização da 
matéria orgânica e disponibilização dos nutrientes e aumento da microbiota no sistema solo (BOECHAT et al., 2013). Porém, são poucos os estudos com este biofertilizante, principalmente sobre a quantidade adequada a se adicionar à substrato para plantas.

A utilização de técnicas não destrutivas para obter informações morfofisiológicas das plantas tem sido amplamente empregada. Os desvios de simetria de órgãos bilaterais verificam a capacidade da planta em conter distúrbios genéticos ou ambientais que ocorram durante $o$ cultivo (SILVA et al., 2011).

$\mathrm{O}$ uso de parâmetros da fluorescência da clorofila- $a$ tem sido difundido, pois avaliam o desempenho qualitativo e quantitativo da absorção e aproveitamento da energia luminosa através do fotossistema II e as relações com a capacidade fotossintética da planta (AZEVEDO NETO et al., 2011). Desta forma, essas características são capazes de predizer distúrbios decorrentes dos tratos culturais, afim de obtenção de mudas de elevada qualidade.

Com base no exposto, objetivou-se verificar o efeito do bokashi nos aspectos morfométricos e fotoquímicos em mudas de C. adamantium.

\section{MATERIAL E MÉTODOS}

Os frutos de $C$. adamantium foram coletados aleatoriamente de matrizes (Autorização de Acesso e de Remessa de Amostra de Componente do Patrimônio Genético $\mathrm{n}^{\mathrm{o}}$ 010220/2015-1 - CNPq/CGEN/MMA) em área remanescente de Cerrado (18 $07^{\prime} 03^{\prime}$ ' $\mathrm{S}, 54^{\circ} 25^{\prime} 07^{\prime}$ 'W, $452 \mathrm{~m}$ ), Dourados - MS, Brasil. Após a coleta, os frutos foram despolpados manualmente, e imediatamente feito o semeio em bandejas de poliestireno expandido de 128 células, preenchidas com substrato Tropstrato ${ }^{\circledR}$. As bandejas foram acondicionadas sob condições de viveiro com tela de sombreamento de $50 \%$, com duas irrigações diárias, utilizando-se aspersores.

Aos 60 dias após a semeadura, realizou-se o transplantio das plântulas para sacos de polietileno de 1,3 L, contendo Latossolo Vermelho distroférrico (SANTOS et al., 2013), de textura argilosa com cinco doses de bokashi (Garden Bokashi ${ }^{\circledR}$ ), incorporadas: $0,5,10,15$ e $20 \mathrm{~g} \mathrm{~kg}^{-1}$ de solo. A unidade experimental constituiu-se de 20 mudas. Utilizou-se o delineamento experimental de blocos casualizados, com quatro repetições.

As mudas foram acondicionadas sob condições de viveiro (22¹1'43.7'S, 5456'08.5”, $458 \mathrm{~m}$ ), com tela de coloração preta com retenção de $50 \%$ do fluxo luminoso e radiação fotossintética média de 820 Lux. Os tratos culturais constituíram-se de irrigações diárias de forma manual, visando manter a capacidade de campo do substrato de $70 \%$, e arranquio de plantas espontâneas quando necessário. Não houve ocorrência de pragas e incidência de doenças.

$\mathrm{O}$ solo apresentou os seguintes atributos químicos (SILVA, 2009): $\mathrm{pH} \mathrm{CaCl}_{2}=6,75 ; \mathrm{P}=5,8 \mathrm{mg} \mathrm{dm}^{-3} ; \mathrm{K}=1,33$ $\mathrm{mmol}_{\mathrm{c}} \mathrm{dm}^{-3} ; \mathrm{Ca}=3,37 \mathrm{mmol}_{\mathrm{c}} \mathrm{dm}^{-3} ; \mathrm{Mg}=4,52 \mathrm{mmol}_{\mathrm{c}} \mathrm{dm}^{-3}$; $\mathrm{H}+\mathrm{Al}=2,30 \mathrm{mmol}_{\mathrm{c}} \mathrm{dm}^{-3} ; \mathrm{SB}=8,56 \mathrm{mmol}_{\mathrm{c}} \mathrm{dm}^{-3} ; \mathrm{V}(\%)=$ 69,2. Já, o bokashi apresentou os seguintes atributos químicos, segundo dados do fabricante: $\mathrm{pH} \mathrm{CaCl}_{2}=6,1 ; \mathrm{N}=34,0 \mathrm{~g} \mathrm{~kg}$ ${ }^{1} ; \mathrm{P}=8,0 \mathrm{~g} \mathrm{~kg}^{-1} ; \mathrm{K}=7,0 \mathrm{~g} \mathrm{~kg}^{-1} ; \mathrm{Ca}=22,0 \mathrm{~g} \mathrm{~kg}^{-1} ; \mathrm{Mg}=5,0 \mathrm{~g}$ $\mathrm{kg}^{-1}$; relação $\mathrm{C} / \mathrm{N}=11 / 1$; carbono orgânico $=400$.

Decorridos 150 dias após o transplantio, foi contabilizado o número de mudas vivas (sobrevivência), e mensuradas as características biométricas: comprimento da parte aérea (distância do coleto até inflexão da folha mais alta), utilizandose régua graduada, diâmetro do coleto, com paquímetro digital ( $\pm 1,0 \mathrm{~cm}$ acima do nível do substrato), número de folhas, contados manualmente. $\mathrm{O}$ índice de clorofila foi determinado por meio do SPAD (Soil Plant Analyzer Development) com clorofilômetro portátil, no período das 8 às $10 \mathrm{~h}$.

As emissões da fluorescência inicial e máxima da clorofila- $a$, foram mensuradas em folhas totalmente expandidas localizadas no terço médio do ramo submetidas por 30 minutos a condições de escuro utilizando-se leaf clips, e as leituras feitas com fluorômetro portátil (OPTI-SCIENCES Chlorophyll Fluorometer, Hudson, USA). A partir dos dados obtidos calculou-se a fluorescência variável $\left(\mathrm{F}_{\mathrm{v}}=\mathrm{F}_{\mathrm{m}}-\mathrm{F}_{0}\right)$, eficiência de conversão de energia absorvida $\left(\mathrm{F}_{\mathrm{v}} / \mathrm{F}_{0}\right)$, rendimento máximo não fotoquímico $\left(\mathrm{F}_{0} / \mathrm{F}_{\mathrm{v}}\right)$, dissipação de energia, taxa relativa de transporte de elétrons.

Foram determinadas as médias do comprimento (CML) e das larguras dos lados esquerdo (L') e direito (L) do limbo das folhas totalmente expandidas (MENEZES SILVA et al., 2011), obtendo-se a largura máxima do limbo (LML) pela soma dos valores de L e L' (Figura 1).

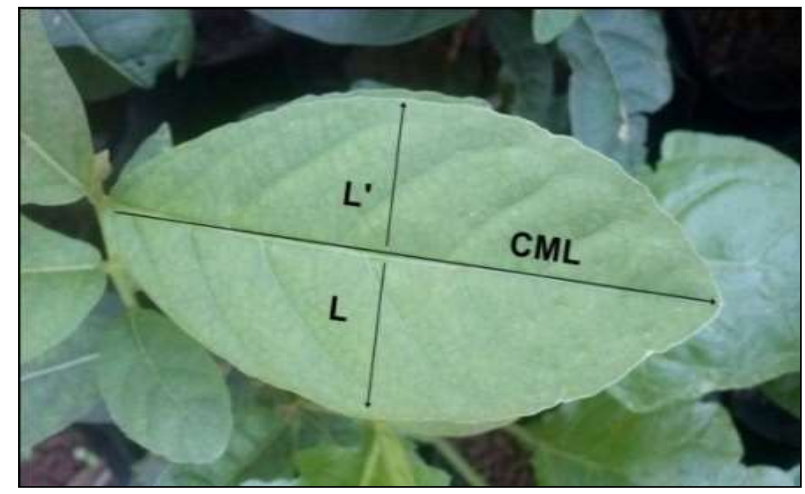

Figura 1. Unidades de amostragem de análises morfométricas foliares em mudas de $C$. adamantium cultivadas com bokashi.

Figure 1. Sampling units of foliar morphometric analyses in seedlings of $C$. adamantium cultivated with bokashi.

Determinaram-se também os desvios de simetria $(d s)$, utilizando metodologia proposta por Cowart e Graham (1999), em que os dados são LOG transformados e normalizados usando transformação Box-Cox $\left[d s=(|d|+0,00005)^{0,33}\right]$; em que $d=\mid \log$ L' - Log L $\mid$ (COWART e GRAHAM, 1999; SOUZA et al., 2005).

Os dados de sobrevivência e desvio de simetria foram transformados em $\sqrt{x}+0,5$ para normalização. Os dados obtidos foram submetidos à análise de variância, e quando significativos a análise de regressão (significância dos modelos matemáticos $)(\mathrm{p} \leq 0,05)$, utilizando-se o software SISVAR (FERREIRA, 2014).

\section{RESULTADOS}

Constatou-se que ocorreu mortalidade de $100 \%$ das mudas produzidas com $20 \mathrm{~g}$ de bokashi, não sendo possível incluir essas na análise estatística nas características morfométricas e fotoquímicas. Os resultados da análise de regressão das características avaliadas encontram-se na tabela 1, observando-se que o número de folhas, fluorescência inicial, taxa de transporte de elétrons, rendimento máximo não fotoquímico, assimetria e desvio de simetria não foram significativos. 
Tabela 1. Equações de regressão das características avaliadas em mudas de $C$. adamantium cultivadas com bokashi.

Table 1. Regression equations of characteristics evaluated in $C$. adamantium seedlings cultivated with bokashi.

\begin{tabular}{|c|c|c|}
\hline Característica & Equação & $\mathrm{R}^{2}$ \\
\hline \multicolumn{3}{|c|}{ Crescimento } \\
\hline $\mathrm{AP}$ & $\hat{y}=\bar{y}=3,52 \mathrm{~cm}$ & S/aj \\
\hline NF & $\hat{\mathrm{y}}=\overline{\mathrm{y}}=4,3$ & Ns \\
\hline \multicolumn{3}{|c|}{ Fluorescências e eficiência fotoquímica } \\
\hline $\mathrm{F}_{0}$ & $\hat{\mathrm{y}}=\overline{\mathrm{y}}=0,215$ elétrons quantum ${ }^{-1}$ & Ns \\
\hline $\mathrm{F}_{\mathrm{m}}$ & $\hat{\mathrm{y}}=\overline{\mathrm{y}}=0,857$ elétrons quantum ${ }^{-1}$ & S/aj \\
\hline $\mathrm{F}_{\mathrm{v}}$ & $\hat{y}=\bar{y}=0,642$ elétrons quantum ${ }^{-1}$ & S/aj \\
\hline $\mathrm{D}_{\mathrm{e}}$ & $\hat{\mathrm{y}}=\overline{\mathrm{y}}=0,142$ elétrons quantum ${ }^{-1}$ & S/aj \\
\hline ETR & $\hat{\mathrm{y}}=\overline{\mathrm{y}}=210,23$ elétrons quantum ${ }^{-1}$ & Ns \\
\hline $\mathrm{F}_{\mathrm{v}} / \mathrm{F}_{\mathrm{m}}$ & $\hat{y}=\bar{y}=0,736$ elétrons quantum ${ }^{-1}$ & S/aj \\
\hline $\mathrm{F}_{0} / \mathrm{F}_{\mathrm{v}}$ & $\hat{\mathrm{y}}=\overline{\mathrm{y}}=0,344$ elétrons quantum ${ }^{-1}$ & $\mathrm{Ns}$ \\
\hline \multicolumn{3}{|c|}{ Morfometria foliar } \\
\hline Assimetria & $\hat{\mathrm{y}}=\overline{\mathrm{y}}=0,458 \mathrm{~cm}$ & $\mathrm{Ns}$ \\
\hline$d s$ & $\hat{\mathrm{y}}=\overline{\mathrm{y}}=0,037$ & $\mathrm{Ns}$ \\
\hline \multicolumn{3}{|c|}{$\begin{array}{l}\mathrm{AP}=\text { altura de plantas; } \mathrm{NF}=\text { número de folhas; } \mathrm{F}_{0}=\text { fluorescência inicial; } \mathrm{F} \\
=\text { fluorescência variável; } \mathrm{F}_{\mathrm{m}}=\text { fluorescência máxima; De = dissipação de } \\
\text { energia; } \mathrm{ETR}=\text { Taxa de transporte de elétrons; } \mathrm{F}_{\mathrm{v}} / \mathrm{F}_{\mathrm{m}}=\text { eficiência fotoquímica } \\
\text { do fotossistema II; } \mathrm{F}_{\mathrm{o}} / \mathrm{F}_{\mathrm{v}}=\text { rendimento máximo não fotoquímico; } \mathrm{S} / \mathrm{aj} .=\text { sem } \\
\text { ajuste; } \mathrm{ns}=\text { não significativo. }\end{array}$} \\
\hline
\end{tabular}

A sobrevivência das mudas reduziu em função das doses crescentes de bokashi (Figura 2a). O diâmetro do coleto máximo $(0,99 \mathrm{~mm})$ foi observado nas mudas cultivadas em solo com adição de 6,48 $\mathrm{g}$ de bokashi (Figura 2b).

Os dados de altura, fluorescência variável e máxima das clorofila- $a$ de mudas de $C$. adamantium não se ajustaram aos modelos matemáticos empregados, obtendo-se médias de 3,52 $\mathrm{cm}, 0,642$ e 0,857 elétrons quantum ${ }^{-1}$, respectivamente (Tabela $1)$.
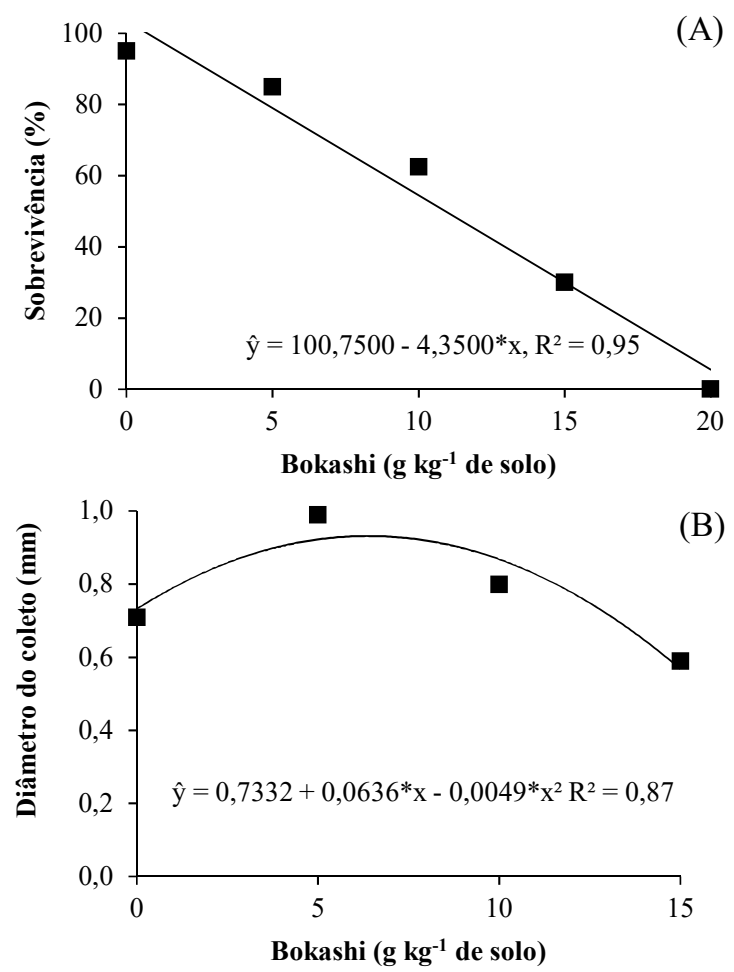

Figura 2. Sobrevivência (A) e diâmetro de coleto (B) em mudas de $C$. adamantium cultivadas com bokashi.

Figure 2. Survival (a) and diameter of collect (B) in seedlings of $C$. adamantium cultivated with bokashi.
O índice de clorofila máximo $(39,07)$ foi observado nas mudas cultivadas sob 4,83 g de bokashi (Figura 3a). Constatou-se redução da $\mathrm{F}_{\mathrm{v}} / \mathrm{F}_{0}$ em função das doses crescentes de bokashi, sendo a maior razão $\left(3,75\right.$ elétrons quantum $\left.{ }^{-1}\right)$ sem a adição de bokashi, enquanto a menor (2,42 elétrons quantum ${ }^{-}$ ${ }^{1}$ ) ocorreu nas plantas cultivadas com $10 \mathrm{~g}$ de bokashi (Figura $3 b)$. Houve aumento nas características morfométricas foliares com doses crescentes de bokashi, em que, o maior CML $(6,47$ $\mathrm{cm})$ e LML (12,90 cm) ocorreram sob $15 \mathrm{~g}$ de bokashi (Figura $4 \mathrm{a}$ e b).
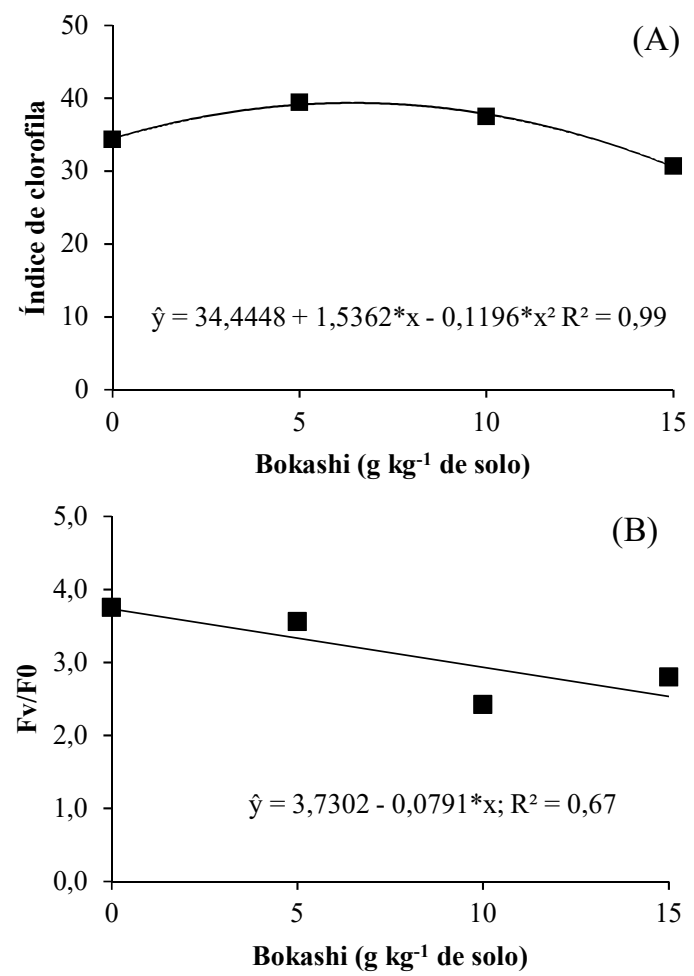

Figura 3. Índice de clorofila (A) e eficiência máxima no processo fotoquímico no FSII (B) em folhas de mudas de C. adamantium cultivadas com bokashi.

Figure 3. Chlorophyll index (a) and energy conversion efficiency absorbed in FSII (B) in leaves of C. adamantium seedlings cultivated with bokashi.

\section{DISCUSSÃO}

A redução da taxa de sobrevivência das mudas de $C$. adamantium com doses crescentes de bokashi deve-se ao excesso de nutrientes. Isso, porque em elevadas quantidades de nutrientes, geralmente ocorre toxidez para as plantas (RAIJ, 2011), principalmente na fase inicial. Estudos sobre quantidade adequadas dos nutrientes são importantes para o desenvolvimento das mudas, pois tanto o excesso como a falta causam desequilíbrios na absorção (COSTA et al., 2012). Resultado semelhante foi observado por Fernandes et al. (2011), no qual, a aplicação de doses crescentes de Fertbokashi $^{\circledR}$, reduziu a sobrevivência de mudas clonais de Eucalyptus urophylla (S.T. Blake), sendo a maior sobrevivência sem a aplicação do bokashi.

O maior diâmetro do coleto observado favorece a estabilização e translocação de fotoassimilados para a muda em formação (CARNEVALI et al., 2015). Costa et al. (2012) estudando a formação de mudas de $C$. adamantium, em diferentes substratos e luminosidade, observaram que as plantas cultivadas em ambiente protegido sombreado e substrato composto de $20 \%$ solo $+80 \%$ de Organosuper $^{\circledR}$, 
obtiveram maior diâmetro do caule e com $100 \%$ de Organosuper $^{\circledR}$ ocorreram os menores diâmetros, podendo evidenciar que doses maiores de composto orgânico e/ou biofertilizantes podem acarretar no excesso de nutrientes, ocasionando toxidade para o desenvolvimento das plantas.
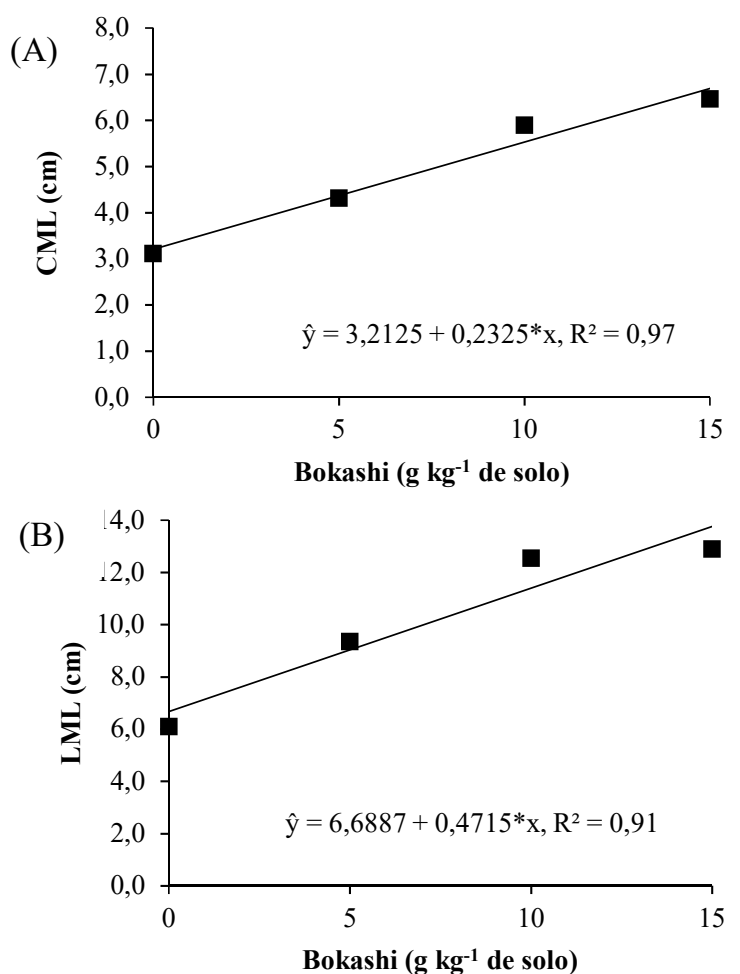

Figura 4. Comprimento (A) e Largura do limbo (B) em mudas de $C$. adamantium com bokashi.

Figure 4. Length (A) and width of Limbo (B) in C. adamantium seedlings with bokashi.

Os dados de altura das mudas não se ajustaram devido ao fato da espécie ser uma planta nativa e apresentar alta diversidade genética, havendo assim, maior variabilidade na obtenção das mudas. Isso porque, alguns autores descreveram que a guavira possui alta variação genética para a altura de plantas, podendo não seguir atributos originais (MIRANDA et al., 2016).

Cabe ressaltar que, mesmo não tendo ajuste, essas características são importantes no processo de transferência de energia, pois a fluorescência variável representa o fluxo de elétrons do centro de reação do FS II até a pastoquinona A (QA), enquanto a fluorescência máxima representa a energia liberada ou perda pelos elétrons, podem alcançar a QA (receptora primária de elétrons do FS II) (BAKER, 2008). O mesmo ocorreu para a eficiência fotoquímica do fotossistema II $\left(\mathrm{F}_{\mathrm{v}} / \mathrm{F}_{\mathrm{m}}\right)$ e dissipação de energia, obtendo médias de $0,736 \mathrm{e}$ 210,23 elétrons quantum ${ }^{-1}$, respectivamente (Tabela 1$)$.

Esses resultados estão diretamente ligados, pois indicadores do processo fotoquímico do FS II, podendo predizer que reduções nos valores indicam que o aparato fotoquímico está debilitado (SUASSUNA et al., 2010). As respostas fotoquímicas das plantas podem variar, ou seja, nem sempre as espécies são influenciadas pelos tratos culturais e condições de estresse. Avaliando-se plantas jovens de $P$. guineense (Myrtaceae) submetidas a doses de alumínio, radiação UV-B ou alagamento do substrato, verificou-se que não influencia desses fatores nas características da fluorescência da clorofila- $a$ (MAURO BARBOSA et al., 2014).

O maior índice de clorofila observado é um bom indicador de $\mathrm{N}$ nas folhas das mudas de C. adamantium; salienta-se que, 50 a $70 \%$ do $\mathrm{N}$ nas folhas encontra-se incorporado às moléculas de clorofila (SILVA et al., 2014), fator interessante tendo em vista que a guavira é exigente em $\mathrm{N}$ e $\mathrm{P}$ para o seu crescimento (VIEIRA et al., 2011). O índice de clorofila foi próximo ao índice médio $(36,0)$ observado por Vieira et al. (2011) em C. adamantium cultivada com adubação de fósforo e nitrogênio. Assim, nota-se a importância da adição de bokashi na fase de produção de mudas.

A redução de $F_{v} / F_{0}$ com doses crescentes de bokashi é indicador da menor eficiência máxima no processo fotoquímico no FSII e/ou da atividade fotossintética potencial (razão máxima de produção quântica dos processos concorrentes fotoquímicos e não fotoquímicos no FSII) (ZANANDREA et al., 2006). Assim, esses resultados levam a compreender perturbações ocasionadas pelos fatores de estudos, tal como observado neste trabalho.

O incremento do CML e LML com aumento de bokashi ao substrato deve-se ao fato de que o bokashi é um biofertilizante rico em nutrientes, como o nitrogênio, fósforo e potássio (SOUZA e RESENDE, 2003), em que esses nutrientes podem contribuir no crescimento dos limbos foliares. Shingo e Ventura (2009) observaram que plantas de Brassica oleracea L. var. acephala cultivadas com bokashi (Garden Bokashi ${ }^{\circledR}$ ) apresentaram comprimento e largura do limbo similar ao utilizar substrato comercial, indicando que o biofertilizante é benéfico para o crescimento do limbo foliar, tornando-se uma alternativa de material a ser adicionado na formulação de substratos para mudas, além de reduzir custos de produção e insumos externos.

\section{CONCLUSÕES}

A adição crescente de bokashi favorece as características morfométricas foliares, mas acima de $15 \mathrm{~g}$ de bokashi é prejudicial na sobrevivência de mudas de $C$. adamantium. Portanto, pode-se utilizar $5 \mathrm{~g}$ de bokashi na formulação de substratos para produção de mudas de $C$. adamantium por favorecer as características fotoquímicas mais viáveis.

\section{AGRADECIMENTOS}

Os autores agradecem ao Conselho Nacional de Desenvolvimento Científico e Tecnológico (CNPq) e Coordenação de Aperfeiçoamento de Pessoal de Nível Superior (CAPES), pela concessão das bolsas, e à Fundação de Apoio ao Desenvolvimento de Ensino, Ciência e Tecnologia do Estado de Mato Grosso do Sul (FUNDECT), pelo apoio financeiro.

\section{REFERÊNCIAS}

BAKER, N. R. Chlorophyll Fluorescence: a probe of photosynthesis in vivo. Annual Review of Plant Biology, Palo Alto, v. 59, n. 1, p. 89-113, 2008. DOI: https://dx.doi.org/10.1146/annurev.arplant.59.032607.092 759

CARNEVALI, T. O.; VIEIRA, M. C.; LUCIANO, A. T.; GONÇALVES, W. V.; RODRIGUES, W. B.; RAMOS, M. B. M. Crescimento inicial de Campomanesia xanthocarpa O. Berg sob diferentes composições de substratos. Revista Brasileira de Plantas Medicinais, 
Botucatu, v. 17, n. 2, p. 316-323, 2015. DOI: http://dx.doi.org/10.1590/1983-084X/12_080

COELHO, S. G.; HAAS, A. P. S.; VON POSER, G. L.; SCHAPOVAL, E. E. S.; ELISABETSKY, E. Ethnopharmacological studies of antimicrobial remedies in south of Brazil. Journal of Ethnopharmacology, Lausanne, v. 90, n. 1, p. 135-143, 2004. DOI: https://dx.doi.org/10.1016/j.jep.2003.09.039

COSTA, E.; SILVA, P. N. L.; JORGE, M. H. A.; FERREIRA, A. F. A. Guavira emergence and seedling production with substrates containing organic compost and soil under different screen environments. Revista Brasileira de Fruticultura, v. 34, n. 4, p. 1289-1293, 2012. DOI: http://dx.doi.org/10.1590/S0100-29452012000400040

COWART, N. M.; GRAHAM, J. H. Within and amongindividual variation in fluctuating asymmetry of leaves in the fig (Ficus carica L.). International Journal of Plant Sciences, Chicago, v. 160, n. 1, p. 116-121, 1999. DOI: https://dx.doi.org/10.1086/314104

FERNANDES, S. J. O.; TITON, M.; SANTANA, R. C.; ANTONINI, L. G.; NOGUEIRA, G. S.; BARROS FILHO, N. F. Sobrevivência e crescimento de mudas clonais de eucalipto em resposta à aplicação de fertilizante orgânico. Cerne, Lavras, v. 17, n. 4, p. 601-606, 2011. DOI: http://dx.doi.org/10.1590/S0104-77602011000400020

HIGASHIKAWA, F. S.; SILVA, C. A.; BETTIOL, W. Chemical and physical properties of organic residues. Revista Brasileira de Ciência do Solo, Viçosa, v. 34, n. 5, p. 1743-1752, 2010. DOI: http://dx.doi.org/10.1590/S0100-06832010000500026

LORENZI, H.; BACHER, L.; LACERDA, M.; SARTORI, S. Frutas brasileiras e exóticas cultivadas (de consumo in natura). São Paulo: Instituto Plantarum, 2006. 640 p.

MACHADO, H.; NAGEM, T. J.; PETERS, V. M.; FONSECA, C. S.; OLIVEIRA, T. T. Flavonoides e seu potencial terapêutico. Boletim do Centro de Biologia da Reprodução, Juiz de Fora, v. 27, n. 1, p. 33-39, 2008.

MAURO BARBOSA, V.; SANTOS NOBREGA, M. A.; SANTIAGO, E. F. Respostas Germinativas de Psidium Guineense Swarts (Myrtaceae) e Plantas Jovens a Múltiplos Fatores de Estresse. Ensaios e Ciência, v. 18, n. 4, p. 173-178, 2014.

MIRANDA, E. A. G. C.; BOAVENTURA-NOVAES, C. R.; BRAGA, R. S.; REIS, E. F.; PINTO, J. F.; TELLES, M. P. Validation of EST-derived microsatellite markers for two Cerrado-endemic Campomanesia (Myrtaceae) species. Genetics and Molecular Research, v. 15, n. 1, p. 15017658-15017658, $2016 . \quad$ DOI: https://dx.doi.org/10.4238/gmr.15017658

PASCOAL, A. C. R. F.; EHRENFRIED, C. A.; LOPEZ, B. G.; ARAUJO, T. M.; PASCOAL, V. D. B.; GILIOLI, R.; ANHÊ, G. F.; RUIZ, A. L. T. G.; CARVALHO, J. E.; STEFANELLO, M. E. A.; SALVADOR, M. J. Antiproliferative activity and induction of apoptosis in PC3 cells by the chalcone cardamonin from Campomanesia adamantium (Myrtaceae) in a bioactivity-guided study. Molecules, v. 19, n. 2, p. 1843-55, 2014. DOI: https://dx.doi.org/10.3390/molecules 19021843

PEREIRA, M. C.; STEFFENS, R. S.; JABLONSKI, A.; HERTZ, P. F.; RIOS, A. O.; VIZZOTTO, M.; FLÔRES, S. H. Characterization and antioxidant potential of Brazilian fruits from the Myrtaceae family. Journal of
Agricultural and Food Chemistry, v. 60, n. 12, p. 30613067, 2012. DOI: https://dx.doi.org/10.1021/jf205263f

RAIJ, B. V. Fertilidade do solo e manejo de nutrientes. Piracicaba: IPNI, 2011. 420 p.

RODRIGUES, V. E. G.; CARVAlHO, D. A. Plantas medicinais no domínio dos cerrados. Lavras: UFLA, 2001. 180 p.

SHINGO, G. Y.; VENTURA, M. U. Produção de couve brassica oleracea L. var. acephala com adubação mineral e orgânica. Semina: Ciências Agrárias, Londrina, v. 30, n. 3, p. 589-584, 2009. DOI: http://dx.doi.org/10.5433/16790359.2009v30n3p589

SILVA, F. C. Manual de análises químicas do solo, plantas e fertilizantes. 2. ed. rev. e aum. Brasília: Embrapa Informação Tecnológica, 2009. 627 p.

SILVA, M. A.; SANTOS, C. M.; VITORINO, H. S.; RHEIN, A. F. Pigmentos fotossintéticos e índice SPAD como descritores de intensidade do estresse por deficiência hídrica em cana-de-açúcar. Bioscience Journal, Uberlândia, v. 30, n. 1, p. 173-181, 2014.

SILVA, P. E. M.; SANTIAGO, E. E.; SILVA, E. M.; SÚAREZ, Y. R.; DALOSO, D. M. Fluorescência da clorofila-a e variação da simetria como ferramentas de investigação de plantas sob estresse. Idesia, Arica, v. 29, n. 3, p. 45-52, 2011. DOI: http://dx.doi.org/10.4067/S0718-34292011000300007

SOUZA, J. L.; RESENDE, P. Manual de horticultura orgânica. Viçosa: Aprenda Fácil, 2003. 564p.

SOUZA, G. M.; VIANA, J. O. F.; OLIVEIRA, R. F. Asymmetrical leaves induced by water deficit show asymmetric photosynthesis in common bean Brazilian. Journal of Plant Physiology, Londrina: v. 17, n. 2, p. 223227, 2005. DOI: http://dx.doi.org/10.1590/S167704202005000200005

SUASSUNA, J. F.; MELO, A. S. D.; SOUSA, M. S. S.; COSTA, F. S.; FERNANDES, P. D.; PEREIRA, V. M.; BRITO, M. E. B. Desenvolvimento e eficiência fotoquímica em mudas de híbrido de maracujazeiro sob lâminas de água. Bioscience Journal, Uberlândia, v. 26, n. 4, p. 566-571, 2010.

VIEIRA, M. C.; PEREZ, V. B.; HEREDIA ZÁRATE N. A.; SANTOS, M. C.; PELlOSO, I. A. O.; PESSOA, S. M. Nitrogênio e fósforo no desenvolvimento inicial da guavira [Campomanesia adamantium (Cambess.) O. Berg] cultivada em vasos. Revista Brasileira de Plantas Medicinais, Botucatu, v. 13, n. 1, p. 542-549, 2011.

WOLFF, W. M.; FLOSS, E. L. Correlação entre teores de nitrogênio e de clorofila na folha com o rendimento de grãos de aveia branca. Ciência Rural, Santa Maria, v. 38, n. $6, \quad$ p. $1510-1515, \quad 2008$. DOI: http://dx.doi.org/10.1590/S0103-84782008000600003

ZACCARDELLI, M; DE NICOLA, F.; VILLECCO, D.; SCOTTI, R. The development and suppressive activity of soil microbial communities under compost amendment. Journal of soil science and plant nutrition, Temuco, v. 13, n. 3, p. 730-742, 2013. DOI: http://dx.doi.org/10.4067/S0718-95162013005000058

ZANANDREA, I.; NASSI, F. L.; TURCHETTO, A. C.; BRAGA, E. J. B.; PETERS, J. A.; BACARI, M. A. Efeito da salinidade sob parâmetros de fluorescência em Phaseolus vulgaris. Revista Brasileira Agrociência, Pelotas, v. 12, n .2, p. 157-161, 2006. 